\title{
New type of pump for the heart-lung bypass system
}

\author{
Nodar Gabriel Khodeli ${ }^{1}$, Otar Sergei Shengelia ${ }^{2}$, Zurab Apollon Chkhaidze ${ }^{1}$, Djumber David \\ Partsakhashvili ${ }^{3}$, Tornike Jemal Sologashvili ${ }^{4}$
}

\footnotetext{
1) Tbilisi State University, Medical Faculty, Clinic Of Heart Surgery, Tbilisi, Georgia

${ }^{2)}$ Department Of Technological Researches Of Philips Corporation, Tbilisi, Georgia

3) Tbilisi State University, Medical Faculty, Scientific-training Center Of Experimental Surgery, Tbilisi, Georgia

4) State Hospital, Open Heart Clinic, Tbilisi, Georgia
}

\section{Summary}

Objective: Complications arising from roller pump usage in modern heart-lung bypass (HLB) systems are widely known. Alternative to roller pumps has existed as various devices. This work is dedicated to developing new, considerably simpler and less expensive pump device for HLB systems, which provides maximally physiological pulsating blood flow.

\begin{abstract}
Material and Method: We used pediatric cardiotomy reservoirs of HLB systems as blood chambers. Two such reservoirs were placed in front of the oxygenator, with their in-flow tubes connected to the venous blood tubes, and the outflow tubes - to the entry to the oxygenator. In each reservoir, 2 air outlets tubes were connected to pneumo-receivers of positive and negative pressure. We filled up one of the reservoirs whith closed of exit blood tube by creating negative pressure in it. Simultaneously, with closed entry blood tube of the second reservoir, we ejected blood from it by created positive pressure. Used external pulsator.
\end{abstract}

Results: In stand testing under re-circulation regime, productivity of the device was 12,5 liters/minute. Pressure on exit blood pump was within 0 to $300 \mathrm{~mm}$.Hg. The pump operating system enabled laminar, as well as pulsating flow of liquid, with frequency of $0-200$ pulses/minute. Research is based on experiments conducted on 14 mongrel dogs weighing $20 \mathrm{~kg}$. Standard methodology was used to cannulate Venae cavae and aorta, and to connect perfusion system to body. Duration of complete HLB reached 2 hours. Systolic pressure was maintained within $120-130 \mathrm{~mm} . \mathrm{Hg}$.; diastolic $-70-80 \mathrm{mmHg}$. Velocity of blood flow in the aorta arc fluctuated within $950-1100 \mathrm{ml} / \mathrm{min}$; in the femoral artery - within $60-80 \mathrm{ml} / \mathrm{min}$.

Conclusion: Preliminary tests of the new pump indicate that its hemodynamic characteristics are maximally approximated to physiological ones. In comparison to modern machines, a perfusion system with new pump type has minimal volume of fill-up and does not damage formative elements of blood.

Keywords: Pulsatille flow, blood pump, perfusion. 


\section{Introduction}

Majority of currently operating systems of artificial blood circulation for heart-lung bypass applies roller pumps as injecting units. ${ }^{(4,7)}$ Though currently these units and their control systems are very well developed, number of authors report on complications in the process of their functioning. Complications, related to the constructional characteristics of the roller pumps declare themselves in a form of homeostasis disorder of various degrees, buffer deviations, centralization of blood circulation, accumulation of sub-oxide products, impairment of entirety of membranes of the blood cells etc. ${ }^{(1,3,10,13)}$ Non-physiological blood flow, caused by the roller pump in the aorta and large vessels is regarded as the major reason for such complications.

Numerous attempts of some authors to replace the roller pumps with other pressurizing devices ${ }^{(2,11)}$ remained within the experimental laboratories. At the same time, there exists the reliable alternative to the roller pumps - the artificial heart ventricles, which form in the arterial bed the flood flow maximally close to the physiological, excluding traumas of the blood cells..$^{(6,12)}$ Ventricular Assist Device are widely applied in clinics as the systems of assisted blood circulation, though they are not used in the HLB machines. Supposedly, this is caused by their high prices.

Development of new, simple and cheap perfusion device for HLB machines and study of their hydrodynamic characteristics in the experiments with animals.

\section{Materials and methods}

As a basis model of the pump we used the device developed in our laboratory. ${ }^{(9)}$ In the pump module of the developed device the blood accumulation and its forcing function was unified in two hermetic transparent reservoirs with rigid walls, with the volume selected for given experimental animal. For simplification of assembling of the experimental pump devices (see Fig. 1) used for relatively large test animals we employed cardiotomic reservoirs of used traditional children's disposable HLB systems, with volume of $1000 \mathrm{ml}$ each.

In the assembly scheme of each perfusion machine two such reservoirs were located before membrane oxigenator on the level of surgical table. Two inlet jets for blood (inner diameter up to $6 \mathrm{~mm}$ ) of each of the reservoirs were connected with each other and supply venous trunk (inner diameter up to $10 \mathrm{~mm}$ ). Outlet jets are connected with each other and the trunk leading to the oxigenator (inner diameter up to $6 \mathrm{~mm}$ ). In each of the reservoirs the jets of the air outlets are connected with the pneumatic receivers of positive and negative pressures with special external valves controlled electronically, included into the pump unit, provided filling of one of the reservoirs, creating negative pressure in it, if the outlet trunk is closed and simultaneously, from the other reservoir there was provided pressurizing, supplying positive pressure with the closed inlet trunk.

In the trunk connecting the outlet of oxigenator with the inlet of arterial filter there was placed the external pulsating unit also included into the pump unit. Electronically controlled pulsating unit, pressing the trunk to certain level, allowed changing of the frequency of contractions, steepness of growth and fall of pressure $(\mathrm{dp} / \mathrm{dt})$ and duration of systole and diastole. In the control system there is also included the unit for control of the level of liquid in the reservoirs and switch of valves, regulating sequence of functions of letdown and pressurizing. For use of the perfusion system on small laboratory animals only the volume of the pump reservoirs and diameters of connecting trunks were changed. Thus, the total volume of system filling was changed.

Monitoring of the pneumatic pressure in the chambers of the reservoirs and pressure of pulsation frequency, volume of blood flow in the connecting trunks and vessels of the biological model was pro-vided by means of the electromagnetic sensors of polygraph "Mingograf-81" - Elema Shonander (Sweden) and flow meter "Nihon Kohden" (Japan). In the experiments with the animals there was also measured the temperature in the rectum and oesophagus and cardiogram was made. Acid-base condition of the blood was measured by means of gas analyzer "Astrup" - Denmark.

\section{Results}

Stand tests were conducted with the purpose of determining of hydrodynamic capacities of the pump device and reliability of its control system. They allowed for identification of basic characteristics of the pump device and the machine as a whole. The system, assembled entirely on the vertical holder allows for maximal approach to the object of perfusion, thus significantly 
reducing the volume of filling. ${ }^{(8)}$ In the closed regime of circulation, with 6-mm connecting trunks capacity of the machine was $8.5 \mathrm{l} / \mathrm{min}$. Pressure on the outlet trunk was regulated within $0-300 \mathrm{mmHg}$ and within the system it mainly was dependent on resistance of oxigenator and arterial filter. In the regime "Stop" levels of the liquids in the pump reservoirs was maintained unchanged and this did not require pressing of the trunks. Pump control system allowed for creation of both, laminar and pulsating flow of the liquid on the outflow trunk.

The perfusion system was tested in the acute experiments (14 experiments) on the mongrel dogs of both sexes, with up to $20 \mathrm{~kg}$ weights, on which, in the conditions of intravenous narcosis and sternotomy, by means of standard techniques, there was conducted cannulation of the vena cavas and aorta. The perfusion system was filled with the blood substitute (total volume of filling was 900-1000 ml of Ringer's solution with 5000 units of heparin), in the recirculation process there were removed the bubbles of air and the system was stopped. Arterial trunk was connected from the filter to the aortal cannula and the inflow, venous trunk - to the triple connection of the venous cannulas. The parallel heart-lung bypass in the direction of blood flow was commenced in one of the pump reservoirs.

After its filling to the conditional mark (corresponding to $700 \mathrm{ml}$ ), flow of venous blood was directed to another reservoir and to the first one, air was supplied
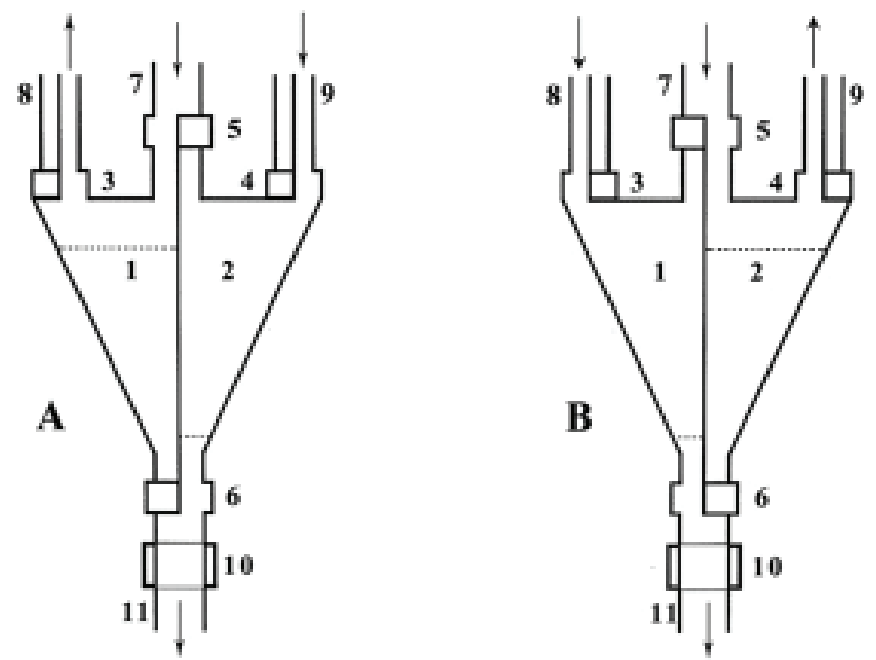

from the receivers under the pressure $(150-200 \mathrm{mmHg}$ ). Thus, the blood pressing through the arterial filter into aorta was commenced. Capacity of the system was no more than $1200 \mathrm{ml} / \mathrm{min}$. Redistribution of blood in the reservoirs, as well as providing pressure and relieve in turns in the chambers was provided automatically from the control system. We conducted simultaneous normothermal perfusion with the operating heart and natural oxygenation for the period up to 1 hour.

Further we provided full HLB, carrying out cardioplegia, clamping aorta and terminating artificial ventilation of lungs. Duration of full heart-lung bypass achieved 3 hours. Systolic pressure was maintained within 120-130 $\mathrm{mmHg}$; diastolic - $70-80 \mathrm{mmHg}$ (average pressure in the femoral artery for entire period of perfusion was no less than $75 \mathrm{mmHg}$ ). Volume velocity of blood flow in the arch of aorta was within $950-1100$ $\mathrm{ml} / \mathrm{min}$ and in the femoral artery $-60-80 \mathrm{ml} / \mathrm{min}$.

Regarding requirements of the experimenters in this sphere $^{(5)}$ there was developed miniature version of the perfusion system, tested in the first experiments on 17 rats, to which it was connected by the scheme: left middle - descending aorta, with retrograde pressurizing of blood (without occlusion of ascending aorta, in the conditions of cold cardioplegia (fibrillation of the ventricles). As a result of full heart-lung bypass in 13 cases there was observed spontaneous restoration of heart operation after 30-minute perfusion. In no one of the conducted experiments hemolysis was not observed in

A-Phase one (Filling of chamber 1 and emptying of chamber 2). Filling and emptying of pump chambers is regulated by a control block so, that as the first chamber of the pump fills with liquid, the second is emptied. In the beginning position, the second chamber is filled with liquid up to the uppermost punctuated line. The occlusive device 3 cutts off the air pressure tube 8 and opens the vacuum tube 8 in chamber 1 . Simultaniously, the occlusive device 4 cuts off the vacuum tube 9 and opens the air pressure tube 9 in chamber 2. In this manner, liquid through tube 7 gets into chamber

1 and fills up gradually up to the punctuated line. The occlusive device 6 of the outlet tube of chamber 1 is closed, while the occlusive device of outlet tube of chamber 2 opens. In this way, liquid from chamber 2, under pressure injected through pulsator into the outlet tube for liquids.

In the second phase (B) the chamber 1 empties while the chamber 2 is filled. Switching of the occlusive devices is controlled by liquid level sensores in chambers.

Figure 1. Principal Scheme and Phases of Pump Operation. 1. First chamber of pump; 2. Second chamber of pump; 3. Air and fluid switching valves; 4. Pulsator; 5. Blood outflow tube from the pmp; 6 . Blood inflow tube in the pump; 7. Tubes for creating air ressure and vacuum; 8. Electrical connections to the control system. 
the blood tests.

\section{Conclusion}

First attempts of operation in the experiments on non-traditional systems of heart-lung bypass and pulsatile perfusion showed possibility of achievement if hemodynamic characteristics identical to physiologi- cal ones. In comparison with the traditional machines the system has minimal volume of filling and does not damage blood cells. Constructive pumping system and entire perfusion system are quite simple and the control system allows achievement of hemodynamic characteristics maximally close to physiological ones. In addition, the system could be applied on the experiments on both, large and small experimental animals.

\section{References}

1. Ashraf S., Bhattacharya K., Zacharias S. et all. Serum S100 release coronary after bypass grafting: roller versus centrifugal pump Ann. Thorac. Surg.-1998.-Vol.66.-N5.-P.1958.

2. Asimakopoulos G., Smith P.L., Ratnatunga C.P., Taylor K.M. Lung injury and acute respiratory distress syndrome after cardiopulmonary bypass Ann. Thorac. Surg.-1999.-Vol. 68.-N3.-P.1107-15.

3. Ballaux P.K., Gourlay T., Ratnatunga C.P., Taylor K.M. A literature review of cardiopulmonary bypass models for rats. Perfusion 1999.Vol.14.- N6, p. 411-7.

4. Chilaia S.M., Khodeli N.G. Biventricular Bypass: Alternative to Univentricular Bypass and Total Artificial Heart-Bridge. Artificial Organs.-1991.-Vol.15.-№5.-P.357-62.

5. Gundry S.R., Romano M.A., Howard Shattuck O. Seven-year followup coronary artery bypasses performed with and without cardiopulmonary bypass. J.Thorac. Cardiovasc. Surg. 1998.- Vol.115.- N6, p.1273.
6. Khodeli N., Chanturia R., Landau I., Mumladze M. The pulsatile flow device for the heart-lung bypass. Statepatent P 2467. Georgia.1999.

7. Khodeli N., Partsakhashvili D. The pulsatile flow device for the heartlung bypass. Statepatent P 3975. Georgia. 2004.

8. McCusker K., Lee S. Post cardiopulmonary bypass bleeding: an intraductory review. J. Extra Corpor Technol.- 1999.-Vol.31.-N1.-P.23-36.

9. Mulay A.V., Zacharias S., Hansbro S.D. Should intraaortic ballon countrpulsation be continued during cardiopulmonary bypass? J. Thorac. Cardiovasc. Surg.-1997.-Vol.114.-N6.-P.1128.

10. Rakhorst G., Hensens A.G., Verkerke G.J. In -vivo evaluation of the "HIA-VAD": a new German ventricular assist device. J.Thorac.Cardiovasc.Surg.1994.- Vol.42.-N3, p.136-40.

11. Sistino J.J., Acsell J.R. Systemic inflammatory response syndrome (SIRS) following emergency cardiopulmonary bypass: a case report and literature review. J. Extra Corpor Technol 1999.- Vol.31.- N1, p.37-43.

Received: 23/11/2012

Accepted: 17/12/2013

Published: 15/09/2014

Disclosure and conflicts of interest:

Conflicts of interest were not reported.

Corresponding author:

Dr. Nodar Gabriel Khodeli

zurab.chkhaidze@tsu.ge 